\title{
Research Article \\ Effect of High-Irradiance Light-Curing on Micromechanical Properties of Resin Cements
}

\author{
Anne Peutzfeldt, Adrian Lussi, and Simon Flury \\ Department of Preventive, Restorative, and Pediatric Dentistry, School of Dental Medicine, University of Bern, \\ Freiburgstrasse 7, 3010 Bern, Switzerland \\ Correspondence should be addressed to Simon Flury; simon.flury@zmk.unibe.ch
}

Received 19 September 2016; Revised 11 November 2016; Accepted 17 November 2016

Academic Editor: Hwa-Liang Leo

Copyright (C) 2016 Anne Peutzfeldt et al. This is an open access article distributed under the Creative Commons Attribution License, which permits unrestricted use, distribution, and reproduction in any medium, provided the original work is properly cited.

\begin{abstract}
This study investigated the influence of light-curing at high irradiances on micromechanical properties of resin cements. Three dualcuring resin cements and a light-curing flowable resin composite were light-cured with an LED curing unit in Standard mode (SM), High Power mode (HPM), or Xtra Power mode (XPM). Maximum irradiances were determined using a MARC PS radiometer, and exposure duration was varied to obtain two or three levels of radiant exposure (SM: 13.2 and $27.2 \mathrm{~J} / \mathrm{cm}^{2} ; \mathrm{HPM}: 15.0$ and $30.4 \mathrm{~J} / \mathrm{cm}^{2}$; XPM: 9.5, 19.3, and $\left.29.7 \mathrm{~J} / \mathrm{cm}^{2}\right)(n=17)$. Vickers hardness $\left(H_{V}\right)$ and indentation modulus $\left(E_{\mathrm{IT}}\right)$ were measured at $15 \mathrm{~min}$ and $1 \mathrm{week}$. Data were analyzed with nonparametric ANOVA, Wilcoxon-Mann-Whitney tests, and Spearman correlation analyses $(\alpha=0.05)$. Irradiation protocol, resin-based material, and storage time and all interactions influenced $H_{V}$ and $E_{\text {IT }}$ significantly $(p \leq 0.0001)$. Statistically significant correlations between radiant exposure and $H_{V}$ or $E_{\mathrm{IT}}$ were found, indicating that high-irradiance lightcuring has no detrimental effect on the polymerization of resin-based materials $(p \leq 0.0021)$. However, one resin cement was sensitive to the combination of irradiance and exposure duration, with high-irradiance light-curing resulting in a $20 \%$ drop in micromechanical properties. The results highlight the importance of manufacturers issuing specific recommendations for the lightcuring procedure of each resin cement.
\end{abstract}

\section{Introduction}

Light-curing resin-based materials currently used in dentistry polymerize as the result of irradiation with visible light in the blue or violet/blue range. The total energy of the irradiation, that is, the radiant exposure $\left(\mathrm{J} / \mathrm{cm}^{2}\right)$, is the product of the irradiance $\left(\mathrm{mW} / \mathrm{cm}^{2}\right)$ and the exposure duration (s), and numerous studies have confirmed that degree of conversion and mechanical properties of the light-cured materials increase with increasing radiant exposure [1-7]. In an effort to reduce costly chair-side time, newly marketed LED (light emitting diode) curing units offer high irradiances to allow reductions in exposure duration. Thus, most modern LED curing units operate at higher irradiances (1000$1500 \mathrm{~mW} / \mathrm{cm}^{2}$ ) than do conventional QTH (quartz-tungstenhalogen) curing units, which might typically have irradiances of $400-500 \mathrm{~mW} / \mathrm{cm}^{2}[8,9]$. One contemporary LED curing unit (VALO, Ultradent) features three different modes of increasing irradiance (according to manufacturer: Standard mode $\approx 1000 \mathrm{~mW} / \mathrm{cm}^{2}$, High Power mode $\approx 1400 \mathrm{~mW} / \mathrm{cm}^{2}$, and Xtra Power mode $\approx 3200 \mathrm{~mW} / \mathrm{cm}^{2}$ ), the highest being much higher than that of QTH curing units and higher than that of many other LED curing units $[9,10]$.

One popular type of light-curing resin-based material is resin cement. These cements are primarily used for luting ceramic restorations and they most often come in dualcuring versions so as to ensure polymerization in regions where light-curing is dubious. Resin cements need to possess superior mechanical properties in order to support the overlying restoration and to avoid abrasion at the restoration margins. Among the mechanical properties, surface microhardness reflects not only the material's resistance to wear and abrasion [11] but for a given resin-based material, surface microhardness also has been shown to be an indirect measure of the degree of conversion of the polymer [12-14].

Because of the great importance of the irradiation procedure for the material properties and clinical success of the resulting restorations, it seems relevant to verify the 
influence of irradiance when applying irradiances that are much higher than those traditionally used. Consequently, the aim of this work was to investigate the influence of relatively high irradiances, as offered by the VALO LED curing unit, on micromechanical properties (Vickers hardness; $H_{V}$ and indentation modulus; $E_{\mathrm{IT}}$ ) of resin cements. As representatives of resin cement one dual-curing, self-etch adhesive resin cement and two dual-curing, self-adhesive resin cements were chosen, and a light-curing, flowable resin composite was included as control. The null hypotheses to be tested were (1) light-curing at high irradiance and reduced exposure duration does not influence the micromechanical properties of the resin-based materials and (2) the micromechanical properties of the resin-based materials do not increase after one week of storage.

\section{Materials and Methods}

The resin-based materials used are listed in Table 1. The dual-curing, self-etch resin cement Panavia F2.0 was handmixed according to the manufacturer's instructions with a 1:1 ratio of paste $A$ and paste $B$. The two dual-curing, self-adhesive resin cements (RelyX Unicem 2 Automix and SpeedCEM Plus) were used with the mixing tips delivered by the manufacturers. Panavia F2.0 and SpeedCEM Plus were stored in the refrigerator and moved to room temperature $1 \mathrm{~h}$ before use.

The resin-based materials were inserted into reusable Teflon molds (diameter: $10 \mathrm{~mm}$; depth: $0.5 \mathrm{~mm}$ ). The material was covered by a Mylar strip (Hawe Stopstrip Straight, KerrHawe SA, Bioggio, Switzerland) and pressed flush with the surface of the mold by means of a glass slide. The material was then irradiated through the Mylar strip at a distance of $0 \mathrm{~mm}$ using a custom-made device to ensure reproducible placement of the tip end of the light-curing unit. Lightcuring was accomplished with a light-emitting diode (LED) VALO curing unit (Ultradent, South Jordan, UT, USA). This light-curing unit offers three modes: Standard mode (SM), High Power mode (HPM), and Xtra Power mode (XPM). The maximum irradiances as well as the spectrum of the VALO curing unit were determined for each of the three modes using a MARC PS radiometer (BlueLight Analytics Inc., Halifax, NS, Canada). The irradiances (mean values and standard deviations of ten measurements per curing mode) and the seven irradiation protocols tested are listed in Table 2 ( $n=17$ per material and protocol). Regardless of the three modes, the spectrum of the VALO curing unit ranged from $380 \mathrm{~nm}$ to $520 \mathrm{~nm}$ with a distinct smaller peak around $405 \mathrm{~nm}$ and a slightly stretched higher peak in the wavelength range of 450 to $470 \mathrm{~nm}$.

\subsection{Measurement of Vickers Hardness $\left(H_{V}\right)$ and Indentation} Modulus $\left(E_{\mathrm{IT}}\right)$. Following light-curing and while still covered with a Mylar strip, each specimen was transferred to a black photo-resistant box to avoid any additional effect of ambient light on the polymerization process. The specimens were kept in the box at $100 \%$ humidity in an incubator at $37^{\circ} \mathrm{C}$ (Memmert UM 500, Schwabach, Germany) for $15 \mathrm{~min}$, allowing time for the autocuring polymerization process of the dual-curing materials to proceed. The first measurement of the micromechanical properties Vickers hardness $\left(H_{V}\right)$ and indentation modulus $\left(E_{\mathrm{IT}}\right)$ were then made, and $H_{V}$ (in $\mathrm{N} / \mathrm{mm}^{2}$ ) and $E_{\mathrm{IT}}$ (in $\mathrm{GPa}$ ) were determined simultaneously with an automatic microhardness indenter device (Fischerscope HM2000, Helmut Fischer GmbH, Sindelfingen, Germany) in analogy to DIN 50359-1:1997-10 [15] and as previously described [16, 17]. All measurements were performed in force-controlled mode for $50 \mathrm{~s}$ with the test load increasing and decreasing between 0.4 and $500 \mathrm{mN}$ at constant speed. The load and the penetration depth of the Vickers indenter (pyramid-shaped diamond, $136^{\circ}$ opening angle) were measured continually during the load-unload hysteresis. $E_{\mathrm{IT}}$ was calculated from the slope of the tangent of the indentation depth curve at maximum force. WIN-HCU software (Helmut Fischer $\mathrm{GmbH}$ ) was used for calculating the micromechanical properties. Five measurements were made on the top surface of each specimen (one central measurement surrounded by four measurements towards the periphery with a distance of $\approx 5 \mathrm{~mm}$ between the four peripheral indentations, each with a distance of $\approx 3 \mathrm{~mm}$ to the central indentation, and resulting in indentation depths of 7.5-20 $\mu \mathrm{m}$ depending on material and irradiation protocol). The five measurements per specimen were averaged and thus $17 H_{V}$ and $17 E_{\text {IT }}$ mean values for each of the four resin-based materials and the seven irradiation protocols were available for statistical analysis. The specimens were once more transferred to black photo-resistant boxes and kept at 100\% humidity in an incubator at $37^{\circ} \mathrm{C}$ (Memmert UM 500) for 1 week before remeasurement of $H_{V}$ and $E_{\mathrm{IT}}$. Thus, 17 new $H_{V}$ and $E_{\mathrm{IT}}$ mean values for each of the four resin-based materials and the seven irradiation protocols were available for statistical analysis.

2.2. Statistical Analysis. Because of lack of normal distribution of the data, nonparametric statistical tests were applied. $H_{V}$ and $E_{\mathrm{IT}}$ values were analyzed with a nonparametric ANOVA according to Higgins [18] to test for significance of the three factors irradiation protocol, storage time, and resin-based material and of interactions, and the $p$ values were corrected with Bonferroni-Holm adjustment for multiple testing. For post hoc tests, WilcoxonMann-Whitney tests were performed without applying further $p$ value adjustment. Spearman rank correlation coefficients were calculated between radiant exposure and single values of $H_{V}$ or $E_{\mathrm{IT}}$ for each resin-based material and each storage time. The significance level was set at $\alpha=0.05$. All statistical analysis was performed with $R$ 3.3.0 (The $R$ Project for Statistical Computing, Vienna, Austria).

A sample size determination from preliminary tests had been performed with NCSS/PASS 2005 (NCSS, Kaysville, UT, USA) under the following conditions: effect size $2 \mathrm{~N} / \mathrm{mm}^{2}$ (as the ability to detect a difference in $H_{V}$ ), power of at least $80 \%$, and level of significance $\alpha=0.05$ and not expecting the median outcome of one group to be higher than that of another group. 
TABLE 1: Resin-based materials used (manufacturers' information).

\begin{tabular}{|c|c|c|}
\hline $\begin{array}{l}\text { Panavia F2.0 (TC) } \\
\text { Kuraray Noritake Dental Inc., Okayama, } \\
\text { Japan }\end{array}$ & \multicolumn{2}{|c|}{$\begin{array}{c}\text { LOT-Nr: AU0119 (Paste A)/BG0054 (Paste B) } \\
\text { Paste/Paste }\end{array}$} \\
\hline Type of resin-based material & \multicolumn{2}{|c|}{ Dual-curing (self-etch) adhesive resin cement } \\
\hline & Paste A & Paste B \\
\hline Methacrylates & \% volume n.a./\% weight n.a. & \% volume n.a./\% weight n.a. \\
\hline Type of methacrylates & $\begin{array}{l}\text { (i) 10-Methacryloyloxydecyl dihydrogen } \\
\text { phosphate (MDP) } \\
\text { (ii) Hydrophobic aromatic dimethacrylate } \\
\text { (iii) Hydrophobic \& hydrophilic aliphatic } \\
\text { dimethacrylate }\end{array}$ & $\begin{array}{l}\text { (i) Hydrophobic aromatic dimethacrylate } \\
\text { (ii) Hydrophobic \& hydrophilic aliphatic } \\
\text { dimethacrylate }\end{array}$ \\
\hline $\begin{array}{l}\text { Initiators, stabilizers, and pigments } \\
\text { Type of initiators }\end{array}$ & $\begin{array}{l}\% \text { volume n.a./\% weight n.a. } \\
\text { (i) dl-Camphorquinone }\end{array}$ & $\begin{array}{l}\% \text { volume n.a./\% weight n.a. } \\
\text { n.a. }\end{array}$ \\
\hline 11 & $\begin{array}{l}\text { (i) Silanated silica } \\
\text { (ii) Silanated colloidal silica }\end{array}$ & $\begin{array}{l}\text { (i) Silanated barium glass } \\
\text { (ii) Surface treated sodium fluoride }\end{array}$ \\
\hline
\end{tabular}

Filler particle size

(i) Total filler content: 59\% volume

RelyX Unicem 2 Automix (A3O)

3M ESPE, Neuss, Germany

$0.04-19 \mu \mathrm{m}$

Type of resin-based material

LOT-Nr: 610318

Paste/Paste (Automix)

\begin{tabular}{lll} 
& \multicolumn{2}{c}{ Dual-curing, self-adhesive resin cement } \\
\hline Methacrylates & Base & Catalyst \\
\hline
\end{tabular}

(i) Triethylene glycol dimethacrylate

(TEGDMA)

Type of methacrylates

(ii) Methacrylate monomers

(iii) Methacrylates with phosphoric acid

groups

Initiators, stabilizers, and pigments

\% volume n.a./\% weight n.a.

Type of initiators

(i) Camphorquinone

\begin{tabular}{lll}
\hline Filler & (i) Glass powder & (i) Silane treated glass \\
(ii) Silane treated silica
\end{tabular}

Filler particle size

(i) Total filler content: $43 \%$ volume

SpeedCEM Plus (Yellow)

$90 \% \sim 12.5 \mu \mathrm{m}$

LOT-Nr: U51719

Ivoclar Vivadent AG, Schaan,

Liechtenstein

Paste/Paste (Automix)

Type of resin-based material Self-adhesive, self-curing resin cement with light-curing option

\begin{tabular}{lll}
\hline & Base & Catalyst \\
\hline Methacrylates & \% volume n.a./\% weight n.a. & \% volume n.a./\% weight n.a.
\end{tabular}

(i) Urethane dimethacrylate (UDMA)

Type of methacrylates

Initiators, stabilizers, and pigments

Type of initiators

Filler

Filler particle size (ii) TEGDMA

(iii) Polyethylene glycol dimethacrylate (PEG-DMA)

(iv) Methacrylated phosphoric acid ester

(v) 1,10-Decandiol dimethacrylate, copolymers n.a.

n.a. n.a.

Dibenzoyl peroxide
$\%$ volume n.a./\% weight n.a.

(i) Ytterbium trifluoride

(ii) Barium glass

(iii) Silicon dioxide

(i) Total filler content: $\sim 45 \%$ volume

0.1-7 $\mu \mathrm{m}$ (mean: $5 \mu \mathrm{m}$ ) 
TABLE 1: Continued.

\begin{tabular}{|c|c|}
\hline $\begin{array}{l}\text { Filtek Supreme XTE Flowable Restorative } \\
\text { (A3) } \\
\text { 3M ESPE, St. Paul, MN, USA }\end{array}$ & $\begin{array}{c}\text { LOT-Nr: N761493 } \\
\text { Paste }\end{array}$ \\
\hline Type of resin-based material & Light-curing, flowable resin composite \\
\hline Methacrylates & \% volume n.a./\% weight n.a. \\
\hline Type of methacrylates & $\begin{array}{l}\text { (i) Bisphenol A-glycidyl methacrylate (Bis-GMA) } \\
\text { (ii) TEGDMA } \\
\text { (iii) Procrylat resins }\end{array}$ \\
\hline Initiators, stabilizers, and pigments & \% volume n.a./\% weight n.a \\
\hline Type of initiators & $\begin{array}{l}\text { (i) Benzotriazol } \\
\text { (ii) Ethyl-4-dimethyl aminobenzoate } \\
\text { (iii) Camphorquinone } \\
\text { (iv) Diphenyliodinium hexafluorophosphate }\end{array}$ \\
\hline Filler & (i) Total filler content: $\sim 46 \%$ volume/ $65 \%$ weight \\
\hline Filler particle size & $\begin{array}{c}\text { (i) Ytterbium trifluoride }(0.1-5 \mu \mathrm{m}) \\
\text { (ii) Nonagglomerated/-aggregated surface modified } 20 \& 75 \mathrm{~nm} \text { silica } \\
\text { (iii) Modified aggregated zirconia }(4-11 \mathrm{~nm}) / \text { silica }(20 \mathrm{~nm}) \text { cluster (average cluster } \\
\text { particle size: } 0.6-10 \mu \mathrm{m})\end{array}$ \\
\hline
\end{tabular}

n.a. = not applicable (no further/detailed information of manufacturer available).

TABLE 2: Irradiances (mean values (standard deviations), $n=10$ ), exposure durations, and resulting radiant exposures of the seven irradiation protocols included.

\begin{tabular}{lccc}
\hline $\begin{array}{l}\text { Irradiation } \\
\text { protocol }\end{array}$ & $\begin{array}{l}\text { Irradiance } \\
\left(\mathrm{mW} / \mathrm{cm}^{2}\right)\end{array}$ & $\begin{array}{c}\text { Exposure duration } \\
(\mathrm{s})\end{array}$ & $\begin{array}{c}\text { Radiant exposure } \\
\left(\mathrm{J} / \mathrm{cm}^{2}\right)\end{array}$ \\
\hline SM 10 & $1324(45.3)$ & 10 & 13.2 \\
SM 20 & $1361(35.8)$ & 20 & 27.2 \\
HPM 8 & $1871(52.2)$ & 8 & 15.0 \\
HPM 16 & $1899(42.4)$ & 16 & 30.4 \\
XPM 3 & $3162(88.2)$ & 3 & 9.5 \\
XPM 6 & $3213(110.9)$ & 6 & 19.3 \\
XPM 9 & $3299(89.0)$ & 9 & 29.7 \\
\hline
\end{tabular}

\section{Results}

The micromechanical properties determined are presented in Table 3 as median, minimum, and maximum values and in Figures 1 and 2 (medians of $H_{V}$ and $E_{\mathrm{IT}}$ ). For $H_{V}$ as well as for $E_{\mathrm{IT}}$, the ANOVA found a statistically significant effect of all three factors irradiation protocol, resin-based material, and storage time and of all interactions $(p \leq 0.0001)$.

As regards irradiation protocol, statistically significant differences in $H_{V}$ and $E_{\mathrm{IT}}$ were found between the protocols for all resin-based materials and at both storage times with the exception of statistically similar 1 week results for RelyX Unicem 2 Automix. The results of the post hoc tests are displayed in Figures 1 and 2. Doubling the exposure duration (and thereby the radiant exposure) resulted in statistically significant increases (1) in $H_{V}$ and $E_{\mathrm{IT}}$ of Panavia F2.0 for all three light-curing modes and both storage times, (2) in $H_{V}$ and $E_{\mathrm{IT}}$ of RelyX Unicem 2 Automix and Filtek Supreme XTE Flowable Restorative for all three light-curing modes at $15 \mathrm{~min}$ and of Filtek Supreme XTE Flowable Restorative in HPM $\left(H_{V}\right)$ and XPM $\left(H_{V}\right.$ and $\left.E_{\mathrm{IT}}\right)$ at 1 week, and finally (3) in $H_{V}$ and $E_{\mathrm{IT}}$ of SpeedCEM for HPM and XPM $\left(H_{V}\right.$ and $\left.E_{\mathrm{IT}}\right)$ at $15 \mathrm{~min}$ and for XPM $\left(H_{V}\right.$ and $\left.E_{\mathrm{IT}}\right)$ at 1 week. Statistically significant correlations between radiant exposure and $H_{V}$ or $E_{\mathrm{IT}}$ were found for all resin-based materials and both storage times $(p \leq 0.0021)$ with the following three exceptions: RelyX Unicem 2 Automix at 1 week $\left(H_{V}\right.$, $\left.p=0.5535 ; E_{\mathrm{IT}}, p=0.5455\right)$ and Filtek Supreme XTE Flowable Restorative $E_{\mathrm{IT}}$ at 1 week $(p=0.1112)$. Indeed, the correlations were generally stronger for the $15 \mathrm{~min}$ results (Spearman correlation coefficients: 0.49-0.87) than for the 1 week results (Spearman correlation coefficients: 0.28-0.72).

As regards resin-based material, all four materials were found to differ significantly from one another $\left(H_{V}: p \leq\right.$ $\left.0.01, E_{\mathrm{IT}}: p \leq 0.0439\right)$ resulting in the following ranking of $H_{V}$ and $E_{\mathrm{IT}}$ (from lowest to highest $H_{V} / E_{\mathrm{IT}}$ ): Panavia F2.0 $\leq$ SpeedCEM < Filtek Supreme XTE Flowable Restorative $<$ RelyX Unicem 2 Automix. As indicated, the ranking of Panavia F 2.0 and SpeedCEM was not clear-cut: in seven out of 28 comparisons there was no significant difference between the two materials (SM $2015 \mathrm{~min}, H_{V}: p=0.4745$; HPM 16 $15 \mathrm{~min}, H_{V}: p=0.1061$; HPM 81 week, $E_{\mathrm{IT}}: p=0.0948$; XPM 31 week, $H_{V}: p=0.1139, E_{\mathrm{IT}}: p=0.1130 ; \mathrm{XPM} 9$ 1 week, $H_{V}: p=0.2485, E_{\mathrm{IT}}: p=0.6098$ ) and in four of the 28 comparisons $H_{V}$ and $E_{\mathrm{IT}}$ of Panavia F2.0 were higher than the corresponding values of SpeedCEM Plus (SM 201 week, $H_{V}$ : $p=0.0004, E_{\mathrm{IT}}: p=0.0001 ; \mathrm{HPM} 161$ week, $H_{V}: p=0.0106$, $\left.E_{\mathrm{IT}}: p=0.0011\right)$.

As regards storage time, storage for 1 week resulted in significantly higher $H_{V}$ values than those obtained after $15 \mathrm{~min}$ for all four resin-based materials and all seven irradiation protocols $(p \leq 0.0006)$. Likewise, $1 \mathrm{wk} E_{\mathrm{IT}}$ values were significantly higher than 15 min values for Panavia F2.0 $(p<0.0001)$ and Filtek Supreme XTE Flowable Restorative 


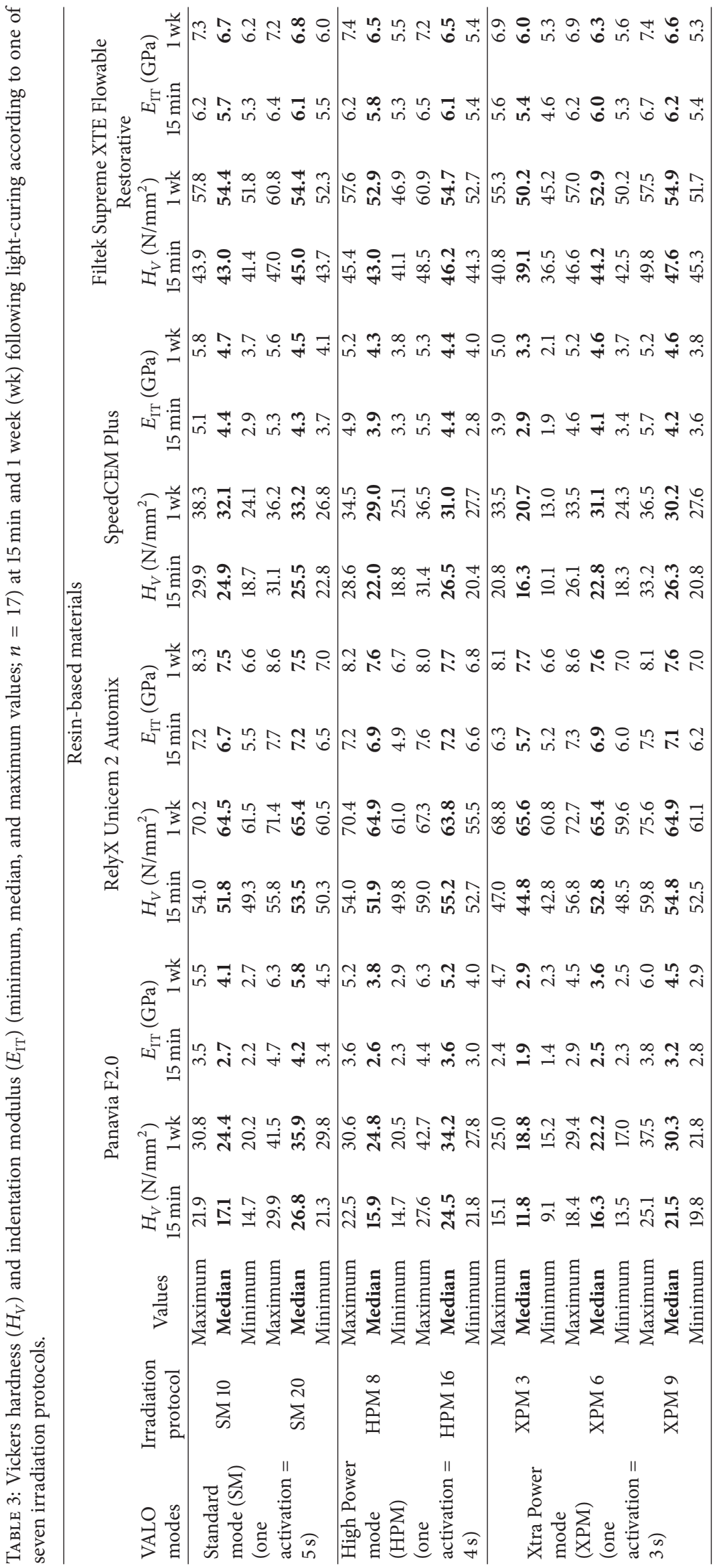




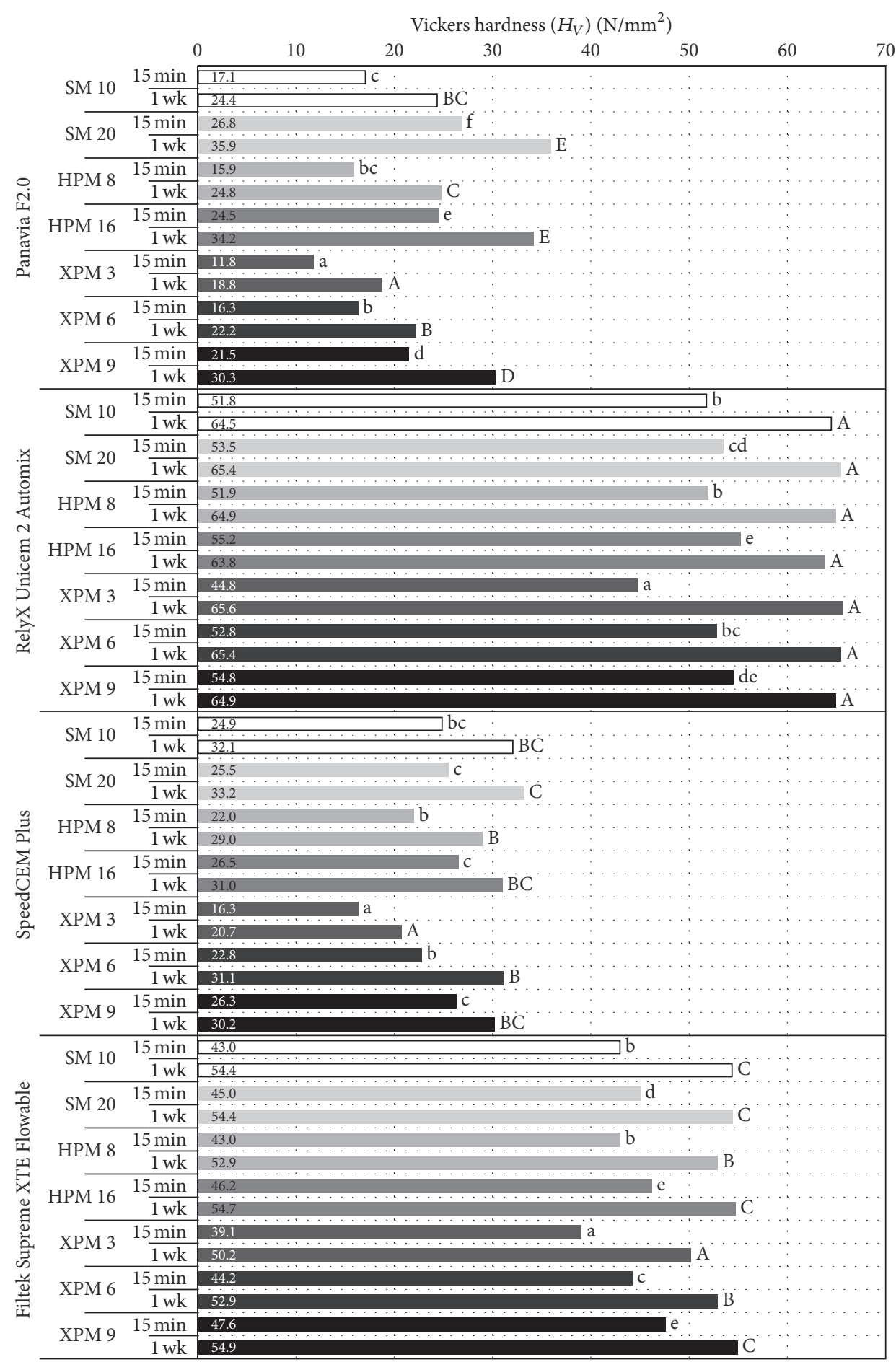

Figure 1: Vickers hardness $\left(H_{V}\right)$ of the four resin-based materials at $15 \mathrm{~min}$ and 1 week (wk) following light-curing according to one of seven irradiation protocols (SM: Standard mode; HPM: High Power mode; XPM: Xtra Power mode). Within each resin-based material, lowercase letters show the results of comparisons between the seven irradiation protocols at 15 min while uppercase letters show the results of comparisons between the seven irradiation protocols at $1 \mathrm{wk}$.

$(p \leq 0.0496)$ regardless of irradiation protocol, for RelyX Unicem 2 Automix $(p \leq 0.0005)$ with one exception (HPM 16, $p=0.0515)$ and for SpeedCEM Plus $(p \leq 0.025)$ with three exceptions (SM 20, $p=0.0537$; HPM 16, $p=$ $0.6542 ; \mathrm{XPM} 9, p=0.1791)$.

\section{Discussion}

The effect of irradiance protocol on the micromechanical properties of resin cements was determined using an automatic microhardness indenter device. The indentation load 


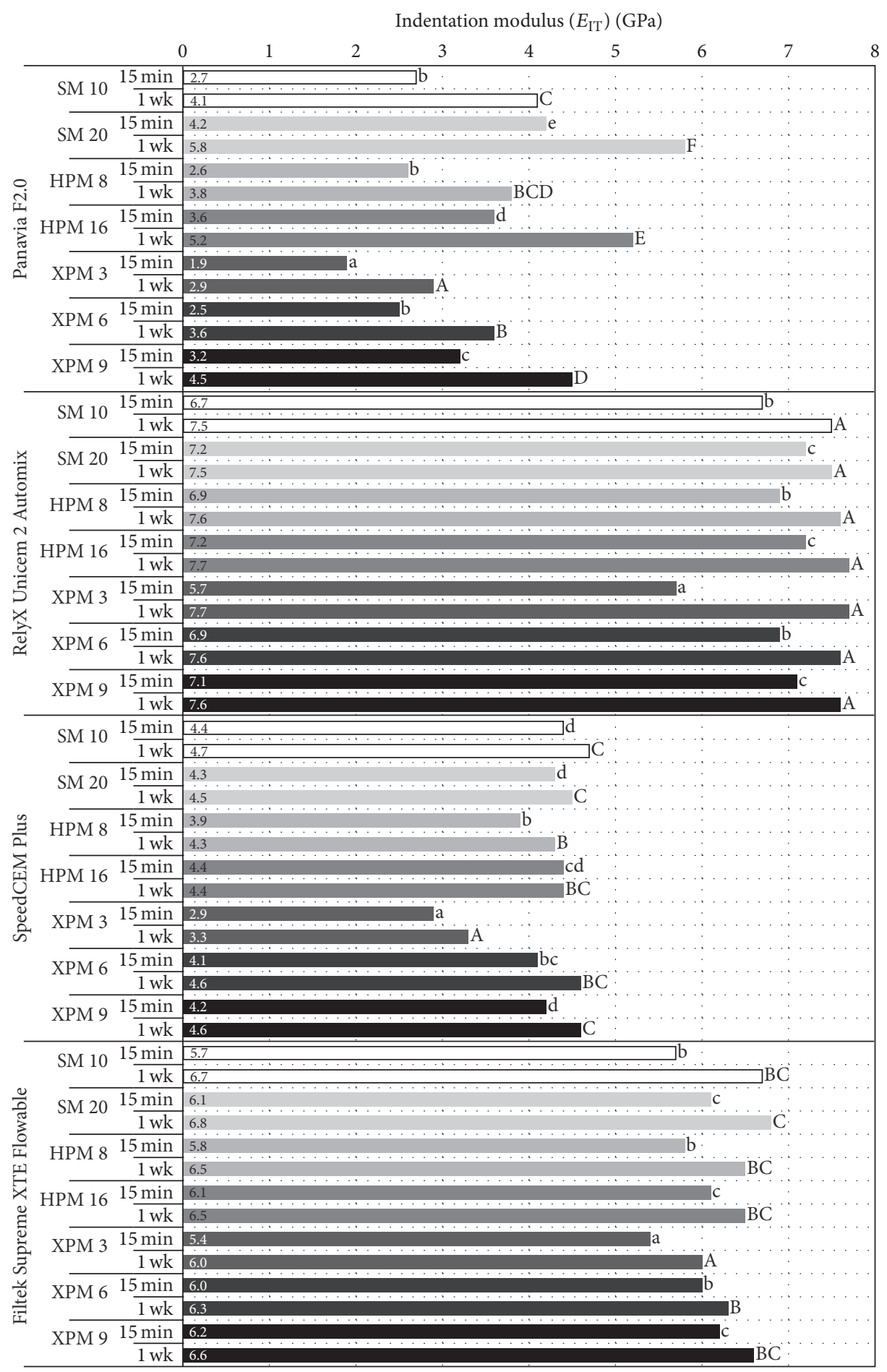

FIGURE 2: Indentation modulus $\left(E_{\mathrm{IT}}\right)$ of the four resin-based materials at $15 \mathrm{~min}$ and 1 week (wk) following light-curing according to one of seven irradiation protocols (SM: Standard mode; HPM: High Power mode; XPM: Xtra Power mode). Within each resin-based material, lowercase letters show the results of comparisons between the seven irradiation protocols at 15 min while uppercase letters show the results of comparisons between the seven irradiation protocols at $1 \mathrm{wk}$.

varied between 0.4 and $500 \mathrm{mN}$ and resulted in indentation depths of $7.5-20 \mu \mathrm{m}$ that were well within the $500 \mu \mathrm{m}$ specimen height. In the clinical situation the resin cements are not exposed to air except at the restoration margins. To avoid any effect of oxygen inhibition, the resin cement specimens were therefore covered with matrices throughout the experimental period.

Within each of the three light-curing modes (SM, HPM, and XPM), doubling the radiant exposure by doubling the exposure duration generally led to significant increases in 
the micromechanical properties. This finding corroborates numerous previous studies on degree of conversion and mechanical properties of not only light-cured, resin-based materials [1-7], but also of dual-curing resin cements [17, 19, 20]. The fact that the dual-curing resin cements reacted similarly to purely light-curing, flowable resin composite reflects that most dual-curing resin cements rely to a large extent on effective light-curing $[10,16,17,21]$. The finding also reflects that dual-curing resin cements seem to contain the same light-curing initiators (most often camphorquinone) as do regular light-curing resin-based materials. However, it is evident from the results of the post hoc analyses that the two resin cements SpeedCEM and, especially, Panavia F2.0 were more influenced by a change in the radiant exposure than were RelyX Unicem 2 Automix and Filtek Supreme XTE Ultra Flowable. Thus, doubling the radiant exposure in each of the three light-curing modes led to a mean increase in micromechanical properties of $40.1 \%$ for Panavia F2.0 while the corresponding increases for RelyX Unicem 2 Automix, SpeedCEM Plus, and Filtek Supreme XTE Flowable Restorative were $5.0 \%, 17.7 \%$, and $5.3 \%$. These results bear witness of a very efficient initiator system present in RelyX Unicem 2 Automix and Filtek Supreme XTE Ultra Flowable. The relative insensitivity of RelyX Unicem 2 is supported by studies on degree of conversion and hardness of RelyX ARC, an etch-and-rinse adhesive resin cement from the same manufacturer that also proved generally insensitive to changes in radiant exposure $[22,23]$. Likewise, the relatively high sensitivity of Panavia F2.0 to changes in radiant exposure is in harmony with previous findings $[17,24]$. These results may be caused by differences in the photoinitiator system indicating poorer efficiency for the latter material.

The main aim of this study was to investigate the effect of irradiation protocols using relatively high irradiances on the micromechanical properties of resin cements, that is, to answer the question "do irradiation protocols that use high irradiance for a short duration yield similar results as do irradiation protocols that use lower irradiance and correspondingly longer durations?" Due to predefined exposure durations of the light-curing unit and because rather short exposure durations were needed, which precluded individual, uncertain timing, radiant exposures were not totally identical for the three different light-curing modes. Consequently, evaluation of the effect of light-curing mode, that is, the specific combination of irradiance and exposure duration at constant radiant exposure, is not straightforward. Overall, there was a linear correlation between radiant exposure and $H_{V}$ or $E_{\mathrm{IT}}$. Thus the "exposure reciprocity law" or "total energy principle" was generally corroborated, dictating that, at a certain radiant exposure, all combinations of irradiance and exposure duration result in comparable material properties, for example, degree of double bond conversion $[2,3,25$, $26]$, hardness $[19,23,27]$, and modulus of elasticity [3, 28, 29]. Despite the general, significant correlation between radiant exposure and micromechanical properties and due to the significant interaction found between resin-based material and irradiation protocol, variation in the light-curing mode did not have the same impact on all four materials. A comparison between the three light-curing modes at the highest radiant exposure (SM $20\left(27.2 \mathrm{~J} / \mathrm{cm}^{2}\right)$; HPM $16\left(30.4 \mathrm{~J} / \mathrm{cm}^{2}\right)$; XPM $\left.9\left(29.7 \mathrm{~J} / \mathrm{cm}^{2}\right)\right)$ suggests that Panavia F2.0 was sensitive to the combination of irradiance and exposure duration. Indeed, high irradiance resulted in a $20 \%$ mean drop in the micromechanical properties (XPM 9 versus SM 20). Other resin-based materials have been found to react negatively to light-curing at very high irradiance and very short durations $[2,3,30-36]$. In their study, comprising a plasma arc curing unit, Feng and Suh [31] explained the lack of reciprocity by the intrinsically higher free radical termination rate when high irradiances are applied for a short time. Later work has shown that whether or not reciprocity is upheld depends on a complex interplay between a multitude of factors such as type and concentration of initiators and viscosity and functionality of monomers as well as degree of conversion [6, 32, 35]. Consequently, the unwillingness of manufacturers to reveal the exact composition of their materials impedes proper analysis and precise explanation of the differences in behavior among the various materials. One possible explanation for the sensitivity of Panavia F2.0 to high-irradiance light-curing could be a relatively low rate and extent of polymerization [21]. In the first stages of polymerization, free radicals are terminated in a bimolecular process. As explained by Feng et al. [35] this implies that many radicals will have a shorter life time during light-curing protocols that use high irradiance and short duration and they will thus react with fewer double bonds before being annihilated. The rate of termination is reduced by immobilization of the free radicals which will prevent their mutual annihilation. At this diffusioncontrolled polymerization stage, termination changes from being a bimolecular process to a monomolecular one. In case of a relatively low rate of polymerization of Panavia F2.0, the transition to monomolecular termination would be retarded and more free radicals would be terminated to result in a lower degree of conversion and in reduced micromechanical properties. As for the two least sensitive materials (RelyX Unicem 2 Automix and Filtek Supreme XTE Flowable Restorative), which are produced by the same manufacturer, they both seem to contain a very sensitive and efficient initiator system for light-curing which would give a high rate of polymerization and an earlier change to monomolecular termination. In the case of Filtek Supreme XTE Ultra Flowable, the efficiency can be explained by the content of diphenyliodonium hexafluorophosphate, a compound known to "boost" the traditional initiator system based on camphorquinone [37]. The fact that light-curing at high irradiance and reduced exposure duration impaired the micromechanical properties of Panavia F2.0 leads to partial rejection of the first hypothesis and highlights the importance of manufacturers issuing detailed recommendations concerning the light-curing procedure and of dentists accurately following these recommendations. It should also be borne in mind that, at the time of light-curing, the resin cement is covered by an indirect, ceramic, or resin composite restoration. When light passes through such restorations, its irradiance decreases due to attenuation. The decrease depends on the type, shade, and thickness of the restorative material and can be quite dramatic. Thus, using the VALO curing unit in High Power mode or Xtra Power mode, the 
irradiance was found to decrease by $\sim 84 \%$ when passing through $1.5 \mathrm{~mm}$ discs of two types of glass ceramic and by $\sim 95 \%$ through $3 \mathrm{~mm}$ discs [10]. Consequently, the negative effect of high irradiance light-curing of Panavia F2.0 may be limited to the resin cement at the restoration margins that are directly exposed to the curing light.

The four resin-based materials displayed significantly different micromechanical properties. Interestingly enough, the materials that were less sensitive to the irradiation protocol (RelyX Unicem 2 Automix and Filtek Supreme XTE Ultra Flowable) were also the materials with the highest Vickers hardness and indentation modulus. They were followed by SpeedCEM and lastly by the most sensitive material Panavia F2.0. The superior micromechanical properties of RelyX Unicem 2 Automix corroborate the findings of a previous study comprising ten dual-curing resin cements [16], and the ranking of the three dual-curing resin cements is in accordance with the ranking found by Flury et al. [17].

Following 1 week of storage, the micromechanical properties had increased for all four resin-based materials, and the second null hypothesis was therefore rejected. The polymerization process of light-cured resin-based materials continues after the end of the irradiation procedure for as long as up to 1 week, continually increasing the degree of conversion of the polymer accompanied by a strengthening of the material [3840]. It is noteworthy that the increase in micromechanical properties from $15 \mathrm{~min}$ to 1 week was higher for Panavia F2.0 than for the three other materials (mean increase: Panavia F2.0, 45\%; Rely Unicem 2 Automix, 19\%, SpeedCEM Plus, 17\%; Filtek Supreme XTE Ultra Flowable, 16\%). The marked postcuring effect of Panavia F2.0 is in agreement with observations in previous studies $[10,17,21]$, the latter work finding an inverse relationship between degree of conversion determined immediately following end of light-curing and the degree of conversion determined after $24 \mathrm{~h}$ [21].

Prior to luting of restorations with Panavia F2.0, tooth surfaces are to be treated with the accompanying self-etching ED Primer II. This primer has been shown to be essential for proper autopolymerization of Panavia F2.0 [10, 41] most likely because of its content of coinitiators [41]. Although the ED Primer II may only be incorporated in the outermost layers of the resin cement, considering the paramount importance of this primer for the polymerization of Panavia F2.0, future experiments should explore whether the ED Primer II has any influence on the sensitivity of the cement to high-irradiance light-curing.

\section{Conclusions}

This study has shown that the irradiation protocol followed when light-curing dual-curing resin cements significantly influences their micromechanical properties. Overall, there was a linear correlation between radiant exposure and $H_{V}$ or $E_{\mathrm{IT}}$, indicating that high-irradiance light-curing has no detrimental effect on the polymerization of resin-based materials. However, one resin cement was negatively affected by light-curing protocols using very short exposure durations at very high irradiances, highlighting the importance of specific recommendations for the light-curing procedure of each product.

\section{Ethical Approval}

This study did not involve human participants and no ethical approval was required.

\section{Disclosure}

The present study was funded by the authors' own institution.

\section{Competing Interests}

The authors declare that they have no conflict of interests, real or perceived, financial or nonfinancial.

\section{Acknowledgments}

The authors would like to thank 3M ESPE, Ivoclar Vivadent, and Kuraray Europe for providing the materials needed. Furthermore, the authors thank G. Fischer, Institute of Mathematical Statistics and Actuarial Science, University of Bern, for the statistical analyses.

\section{References}

[1] A. U. Yap and C. Seneviratne, "Influence of light energy density on effectiveness of composite cure," Operative Dentistry, vol. 26, no. 5, pp. 460-466, 2001.

[2] R. H. Halvorson, R. L. Erickson, and C. L. Davidson, "Energy dependent polymerization of resin-based composite," Dental Materials, vol. 18, no. 6, pp. 463-469, 2002.

[3] A. Peutzfeldt and E. Asmussen, "Resin composite properties and energy density of light cure," Journal of Dental Research, vol. 84, no. 7, pp. 659-662, 2005.

[4] A. R. Benetti, E. Asmussen, E. C. Munksgaard et al., "Softening and elution of monomers in ethanol," Dental Materials, vol. 25, no. 8, pp. 1007-1013, 2009.

[5] M. Dewaele, E. Asmussen, A. Peutzfeldt et al., "Influence of curing protocol on selected properties of light-curing polymers: degree of conversion, volume contraction, elastic modulus, and glass transition temperature," Dental Materials, vol. 25, no. 12, pp. 1576-1584, 2009.

[6] J. G. Leprince, M. Hadis, A. C. Shortall et al., "Photoinitiator type and applicability of exposure reciprocity law in filled and unfilled photoactive resins," Dental Materials, vol. 27, no. 2, pp. 157-164, 2011.

[7] A. C. Shortall, W. El-Mahy, D. Stewardson, O. Addison, and W. Palin, "Initial fracture resistance and curing temperature rise of ten contemporary resin-based composites with increasing radiant exposure," Journal of Dentistry, vol. 41, no. 5, pp. 455463, 2013.

[8] F. A. Rueggeberg, "State-of-the-art: dental photocuring-a review," Dental Materials, vol. 27, no. 1, pp. 39-52, 2011.

[9] K. D. Jandt and R. W. Mills, "A brief history of LED photopolymerization," Dental Materials, vol. 29, no. 6, pp. 605-617, 2013.

[10] S. Flury, A. Lussi, R. Hickel, and N. Ilie, "Light curing through glass ceramics with a second- and a third-generation LED 
curing unit: effect of curing mode on the degree of conversion of dual-curing resin cements," Clinical Oral Investigations, vol. 17, no. 9, pp. 2127-2137, 2013.

[11] K. Kawai, Y. Iwami, and S. Ebisu, "Effect of resin monomer composition on toothbrush wear resistance," Journal of Oral Rehabilitation, vol. 25, no. 4, pp. 264-268, 1998.

[12] Y. L. Yan, Y. K. Kim, K.-H. Kim, and T.-Y. Kwon, "Changes in degree of conversion and microhardness of dental resin cements," Operative Dentistry, vol. 35, no. 2, pp. 203-210, 2010.

[13] D. C. Watts, "Reaction kinetics and mechanics in photopolymerised networks," Dental Materials, vol. 21, no. 1, pp. 2735, 2005.

[14] M. R. Bouschlicher, F. A. Rueggeberg, and B. M. Wilson, "Correlation of bottom-to-top surface microhardness and conversion ratios for a variety of resin composite compositions," Operative Dentistry, vol. 29, no. 6, pp. 698-704, 2004.

[15] EN ISO 14577-1, "Metallic materials-instrumented indentation test for hardness and materials parameters," Part 1: test method, 2002.

[16] N. Ilie and A. Simon, "Effect of curing mode on the micro-mechanical properties of dual-cured self-adhesive resin cements," Clinical Oral Investigations, vol. 16, no. 2, pp. 505-512, 2012.

[17] S. Flury, A. Lussi, R. Hickel, and N. Ilie, "Light curing through glass ceramics: effect of curing mode on micromechanical properties of dual-curing resin cements," Clinical Oral Investigations, vol. 18, no. 3, pp. 809-818, 2014.

[18] J. J. Higgins, Introduction to Modern Nonparametric Statistics, Duxbury Press, Pacific Grove, Calif, USA, 2004.

[19] E. Piva, L. Correr-Sobrinho, M. A. C. Sinhoreti, S. Consani, F. F. Demarco, and J. M. Powers, "Influence of energy density of different light sources on Knoop hardness of a dual-cured resin cement," Journal of Applied Oral Science, vol. 16, no. 3, pp. 189193, 2008.

[20] H. Watanabe, R. Kazama, T. Asai et al., "Efficiency of dualcured resin cement polymerization induced by high-intensity led curing units through ceramic material," Operative Dentistry, vol. 40, no. 2, pp. 153-162, 2015.

[21] S. G. Pereira, R. Fulgêncio, T. G. Nunes, M. Toledano, R. Osorio, and R. M. Carvalho, "Effect of curing protocol on the polymerization of dual-cured resin cements," Dental Materials, vol. 26, no. 7, pp. 710-718, 2010.

[22] M. d. Mainardi, M. C. Giorgi, D. A. Lima et al., "Effect of energy density and delay time on the degree of conversion and Knoop microhardness of a dual resin cement," Journal of Investigative and Clinical Dentistry, vol. 6, no. 1, pp. 53-58, 2015.

[23] P. C. P. Komori, A. B. Paula, A. A. Martin, R. N. Tango, M. A. C. Sinhoreti, and L. Correr-Sobrinho, "Effect of light energy density on conversion degree and hardness of dual-cured resin cement," Operative Dentistry, vol. 35, no. 1, pp. 120-124, 2010.

[24] C. Takubo, G. Yasuda, R. Murayama et al., "Influence of power density and primer application on polymerization of dualcured resin cements monitored by ultrasonic measurement," European Journal of Oral Sciences, vol. 118, no. 4, pp. 417-422, 2010.

[25] N. Emami and K.-J. M. Söderholm, "How light irradiance and curing time affect monomer conversion in light-cured resin composites," European Journal of Oral Sciences, vol. 111, no. 6, pp. 536-542, 2003.

[26] R. Nomoto, K. Uchida, and T. Hirasawa, "Effect of light intensity on polymerization of light-cured composite resins," Dental Materials Journal, vol. 13, no. 2, pp. 198-205, 1994.
[27] R. B. T. Price, C. A. Felix, and P. Andreou, "Effects of resin composite composition and irradiation distance on the performance of curing lights," Biomaterials, vol. 25, no. 18, pp. 4465-4477, 2004.

[28] N. Emami, K.-J. M. Söderholm, and L. A. Berglund, "Effect of light power density variations on bulk curing properties of dental composites," Journal of Dentistry, vol. 31, no. 3, pp. 189196, 2003.

[29] R. L. Sakaguchi and J. L. Ferracane, "Effect of light power density on development of elastic modulus of a model lightactivated composite during polymerization," Journal of Esthetic and Restorative Dentistry, vol. 13, no. 2, pp. 121-130, 2001.

[30] L. Musanje and B. W. Darvell, "Polymerization of resin composite restorative materials: exposure reciprocity," Dental Materials, vol. 19, no. 6, pp. 531-541, 2003.

[31] L. Feng and B. I. Suh, "Exposure reciprocity law in photopolymerization of multi-functional acrylates and methacrylates," Macromolecular Chemistry and Physics, vol. 208, no. 3, pp. 295306, 2007.

[32] M. Hadis, J. G. Leprince, A. C. Shortall, J. Devaux, G. Leloup, and W. M. Palin, "High irradiance curing and anomalies of exposure reciprocity law in resin-based materials," Journal of Dentistry, vol. 39, no. 8, pp. 549-557, 2011.

[33] N. Ilie, A. Kessler, and J. Durner, "Influence of various irradiation processes on the mechanics," Journal of Dentistry, vol. 41, no. 8, pp. 695-702, 2013.

[34] M. C. C. Giorgi, V. Pistor, R. S. Mauler, D. A. N. L. Lima, G. M. Marchi, and F. H. B. Aguiar, "Influence of lightactivation protocol on methacrylate resin-composite evaluated by dynamic mechanical analysis and degree of conversion," Lasers in Medical Science, vol. 30, no. 4, pp. 1219-1223, 2015.

[35] L. Feng, R. Carvalho, and B. I. Suh, "Insufficient cure under the condition of high irradiance and short irradiation time," Dental Materials, vol. 25, no. 3, pp. 283-289, 2009.

[36] D. Selig, T. Haenel, B. Hausnerová et al., "Examining exposure reciprocity in a resin based composite using high irradiance levels and real-time degree of conversion values," Dental Materials, vol. 31, no. 5, pp. 583-593, 2015.

[37] L. S. Gonçalves, R. R. Moraes, F. A. Ogliari, L. Boaro, R. R. Braga, and S. Consani, "Improved polymerization efficiency of methacrylate-based cements containing an iodonium salt," Dental Materials, vol. 29, no. 12, pp. 1251-1255, 2013.

[38] D. C. Watts, O. M. Amer, and E. C. Combe, "Surface hardness development in light-cured composites," Dental Materials, vol. 3, no. 5, pp. 265-269, 1987.

[39] R. Z. Alshali, N. Silikas, and J. D. Satterthwaite, "Degree of conversion of bulk-fill compared to conventional resincomposites at two time intervals," Dental Materials, vol. 29, no. 9, pp. e213-e217, 2013.

[40] R. Pilo and H. S. Cardash, "Post-irradiation polymerization of different anterior and posterior visible light-activated resin composites," Dental Materials, vol. 8, no. 5, pp. 299-304, 1992.

[41] A. L. Faria-e-Silva, R. R. Moraes, F. A. Ogliari, E. Piva, and L. R. M. Martins, "Panavia F: the role of the primer," Journal of Oral Science, vol. 51, no. 2, pp. 255-259, 2009. 

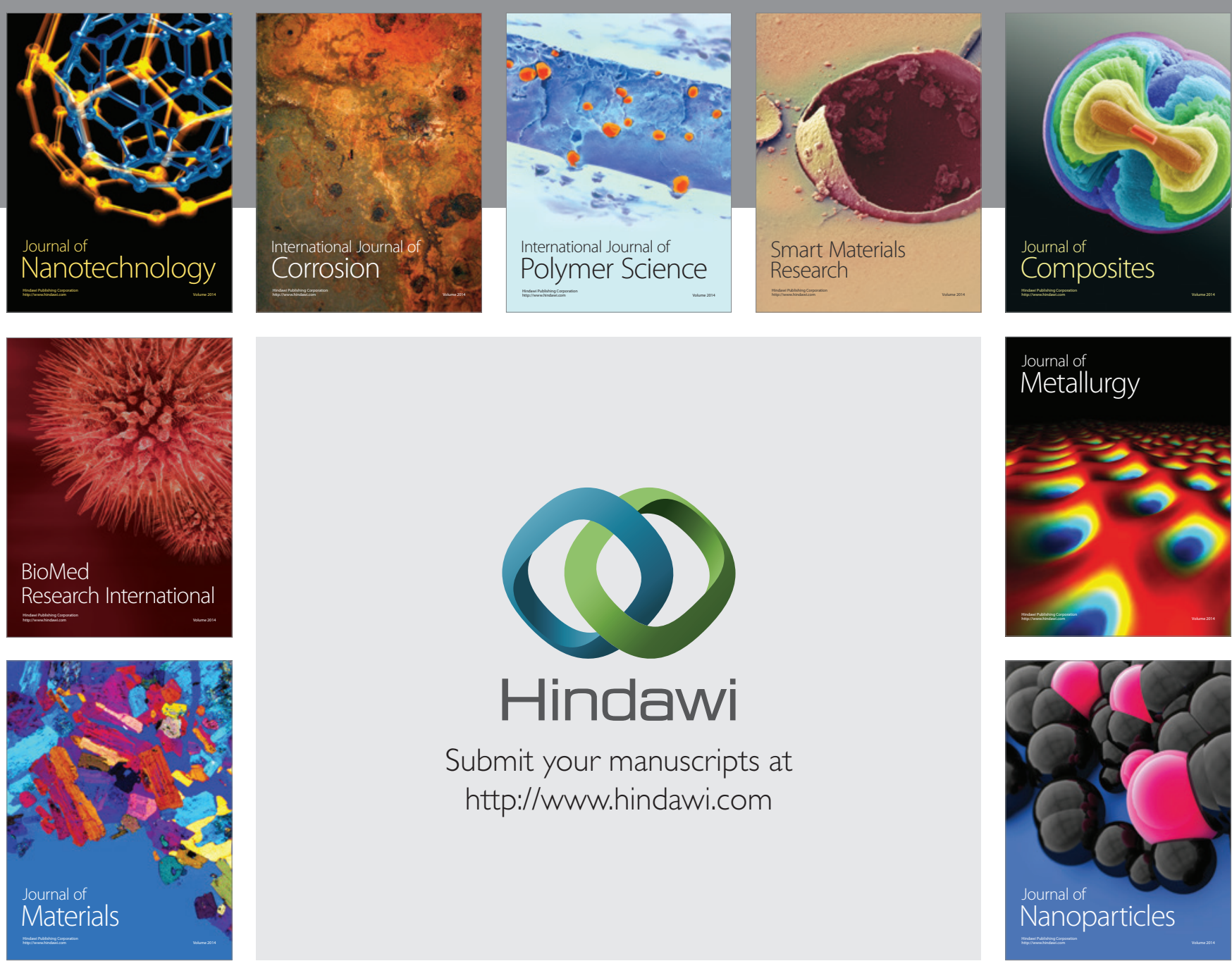

\section{Hindawi}

Submit your manuscripts at

http://www.hindawi.com

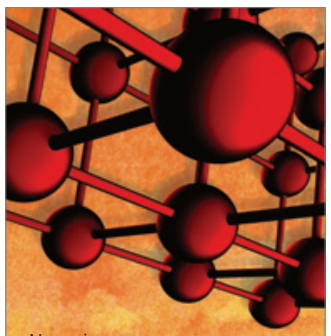

Materials Science and Engineering
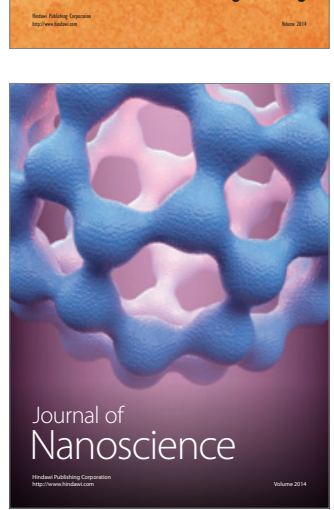
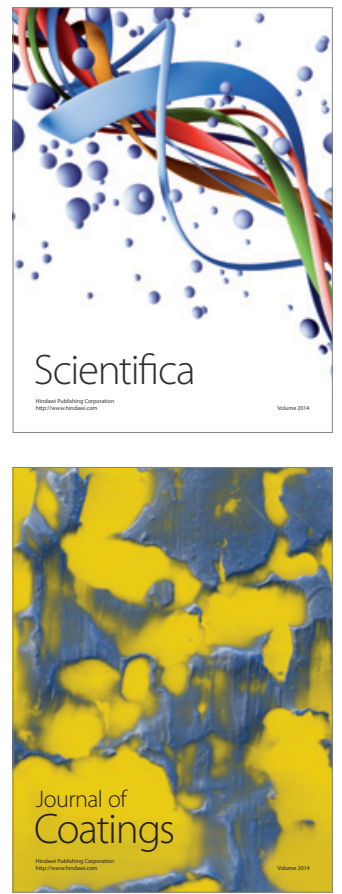
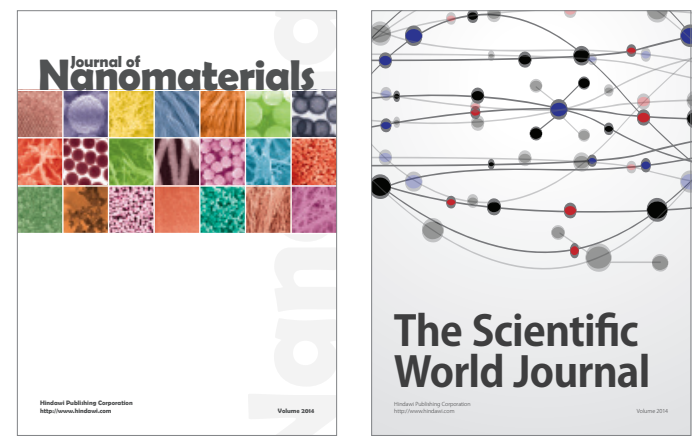

The Scientific World Journal
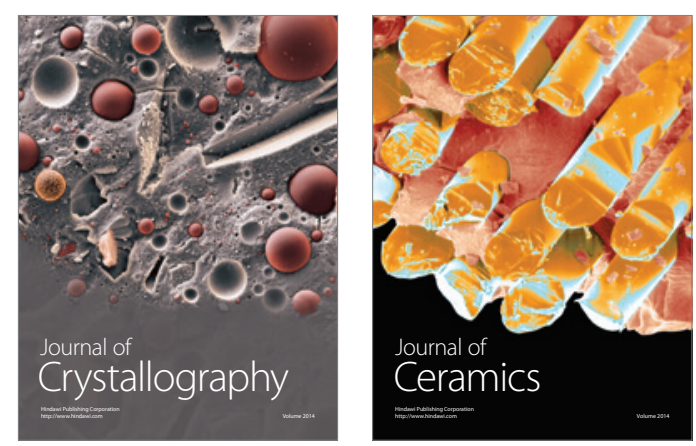
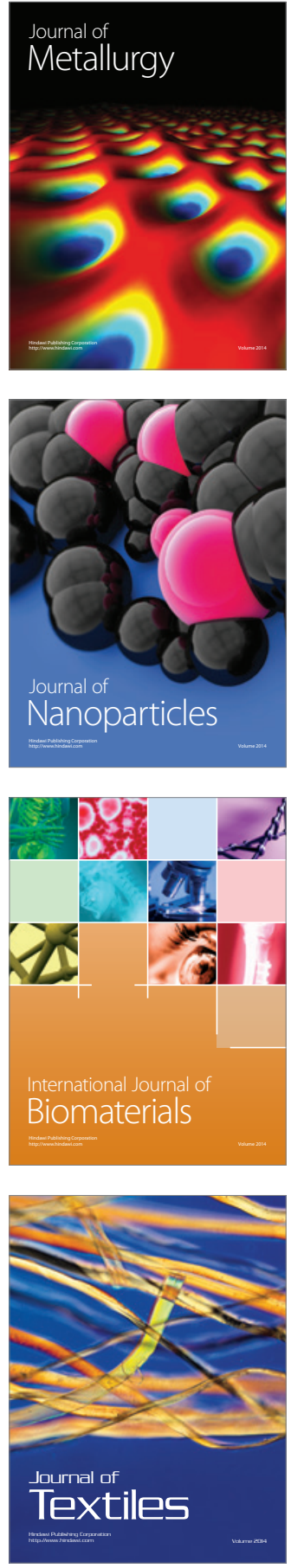